Pacific Journal of Mathematic 


\title{
DECOMPOSITIONS OF HOMOGENEOUS CONTINUA
}

\author{
JAMES T. ROGERS, JR.
}

\begin{abstract}
The purpose of this paper is to present a general theory of decomposition of homogeneous spaces.
\end{abstract}

F. Burton Jones was the first to study properties of homogeneous continua by means of decompositions. In 1951, he showed [12] that a hereditarily unicoherent, homogeneous curve must be indecomposable. His proof used an aposyndetic decomposition.

Soon after, he [11] extended the result to the Jones Aposyndetic Decomposition Theorem. For the case of a decomposable planar continuum $M$, the theorem states that $M$ admits a continuous decomposition such that the decomposition space is a simple closed curve and each element of the decomposition is an indecomposable, homogeneous, acyclic continuum. Recently, the author [13] has improved Jones' theorem in the nonplanar case (the improved version is stated in this paper as Theorem 2).

R. H. Bing [1] proved that the pseudo-arc is homogeneous. Because of Jones' theorem, it would have been natural to ask if there were a planar continuum that admitted a decomposition into pseudo-arcs with the quotient space being a simple closed curve. Such an example was obtained independently by Bing and by Jones in 1954 [3].

C. L. Hagopian and the author [9] have proved that any nonplanar, homogeneous, circle-like continuum that is not a solenoid admits a continuous decomposition into pseudo-arcs such that the quotient space is a solenoid. The author [14] has constructed such a continuum for each solenoid.

Jones [10] and Hagopian [6 and 7] have used decompositions of a proper subcontinuum to investigate properties of homogeneous, plane continua.

David Bellamy and Lewis Lum have communicated to the author that they will use decompositions to prove that a uniquely-arcwise connected continuum cannot be homogeneous.

Clearly, decompositions are a powerful and frequently-used tool in investigating homogeneous continua.

The purpose of this paper is to present a general theory of decomposition of homogeneous spaces. All the decompositions in the theorems above fit into this theory. Theorem 1 presents a special (absolute) case of the theory, easy to state and with enough strength to imply most of the decompositions for curves. We derive through 
it, for instance, the Jones Aposyndetic Decomposition Theorem for curves. We also prove via this decomposition method the new result that an atriodic homogeneous curve containing an arc is a solenoid.

In $\S 2$, we state the general (relative) version of the theory and indicate how a result of Hagopian [6] may be viewed as a consequence of it.

We will use the following version of a theorem of Effros [5].

THEOREM. If $M$ is a homogeneous compactum and $\varepsilon>0$, then there exists a positive number $\delta$ such that if there exists points $x$ and $y$ of $M$ with $d(x, y)<\delta$, then there exists an $\varepsilon$-homeomorphism $h: M \rightarrow M$ such that $h(x)=y$.

The number $\delta$ will be called an Effros number for $\varepsilon$. Recall that $h$ is an $\varepsilon$-homeomorphism if $d(z, h(z))<\varepsilon$, for all $z$ in $M$.

Important also is a result of David Wilson [15], which will be used to show that the elements of certain decompositions are acyclic.

1. Decompositions of homogeneous curves. A curve is a one-dimensional continuum.

THEOREM 1. Let $\mathscr{G}$ be a partition into proper subcontinua of the homogeneous curve $M$. Suppose the homeomorphism group $H$ of $M$. satisfies the following conditions:

$$
\begin{aligned}
& \text { (*) } \text { If } G, G^{\prime} \in \mathscr{Y}, h \in H, \text { then } \\
& \\
& h(G) \cap G^{\prime} \neq \phi=h(G)=G^{\prime} .
\end{aligned}
$$

Then

(1) The partition $\mathscr{G}$ is a continuous decomposition of $M$,

(2) If $G, G^{\prime} \in \mathscr{G}$, then $G$ is homeomorphic to $G^{\prime}$

(3) Each $G$ in $\mathscr{G}$ is a homogeneous, acyclic, hereditarily unicoherent, indecomposable continuum, and

(4) The quotient space $N$ is a homogeneous curve.

Proof. Let $G$ be an arbitrary element of $\mathscr{S}$, and let $U$ be an arbitrary open set of $M$ containing $G$. To show $\mathscr{G}$ is upper semicontinuous, we must exhibit an open set $V$ containing $G$ and lying in $U$ such that every element of $\mathscr{G}$ that intersects $V$ lies in $U$.

Let $\varepsilon=d(G, M-U)$. Choose an Effros number $\delta$ for $\varepsilon$. Let $B$ be the $\delta$-neighborhood of $G$. Let $G^{\prime}$ be an element of $\mathscr{G}$, and let $y$ be a point of $G^{\prime} \cap V$. Let $x$ be a point of $G$ such that $d(x, y)<$ $\delta$. Let $h$ be an $\varepsilon$-homeomorphism of $M$. such that $h(x)=y$. It follows from $\left(^{*}\right)$ that $h(G)=G^{\prime}$. Since $h$ moves no point more than 
$\varepsilon, G^{\prime} \subset U$.

The upper semicontinuous decomposition $\mathscr{G}$ is continuous somewhere and hence, by homogeneity, continuous everywhere.

An alternate proof of the continuity of the decomposition $\mathscr{G}$, using limit inferior and limit superior, may be patterned after an argument of Hagopian [7, Lemma 5].

That the elements of $\mathscr{G}$ are mutually homeomorphic follows from $\left(^{*}\right)$, as does the fact that each element of $\mathscr{G}$ is homogeneous.

We outline a proof that each $G$ in $\mathscr{G}$ is a hereditarily unicoherent, indecomposable continuum and refer the reader to [13, Theorem 3] for a detailed proof exactly applicable to this case. The quotient map of $M$ onto the quotient space $N$ is completely regular. David Wilson [15, Theorem 1] has shown that, in such a case, $H^{1}(G)=0$. Acyclic curves are hereditarily unicoherent. F. B. Jones [12] has shown that homogeneous, hereditarily unicoherent continua are indecomposable.

To prove $N$ is homogeneous is straightforward. Since monotone maps with acyclic point-inverses cannot raise the dimension of a curve, the continuum $N$ is a curve.

This completes the proof of Theorem 1 .

We cite two applications of this theorem. The first result is well-known; the second is new.

Let $x$ and $y$ be points of the continuum $M$. If $M$ contains an open set $G$ and a continuum $H$ such that $x \in G \subset H \subset M-\{y\}$, then $M$ is said to be aposyndetic at $x$ with respect to $y$. If $M$ is aposyndetic at each of its points with respect to every other point, then $M$ is said to be aposyndetic.

The set $L_{x}$ has as members the point $x$ together with all points $z$ of $M$ such that $M$ is not aposyndetic at $z$ with respect to $x$. Each set $L_{x}$ is a continuum. If $M$ is indecomposable, then $L_{x}=M$, for all $x$ in $M$. If $M$ is decomposable, then we have the following version of the Jones Aposyndetic Decomposition Theorem [13].

THEOREM 2. If $M$ is a decomposable, homogeneous continuum, then the collection $\mathscr{G}=\left\{L_{x}: x \in M\right\}$ is a partition of $M$ satisfying condition (*). Hence $\mathscr{G}$ satisfies the conclusion of Theorem 1.

The proof that $\mathscr{G}$ is a partition of $M$ satisfying $\left(^{*}\right)$ may be found in [11].

Bing [2] has shown that a simple closed curve is the only homogeneous plane continuum that contains an arc. The reader may find it interesting to compare the arguments of the next theorem with those of [2].

A continuum is a solenoid if it is homeomorphic to an inverse 
limit of circles with covering maps as the bonding maps. Note that a simple closed curve is a solenoid.

THEOREM 3. If the atriodic, homogeneous curve $M$ contains an arc, then $M$ is a solenoid.

Proof. Suppose $M$ is not a simple closed curve. Let $A$ be an arc component of $M$. We will prove the theorem by listing enough properties of $\bar{A}$ to imply the theorem.

Property 1. If $p$ is a point of $\bar{A}$ and $A_{p}$ is the arc component containing $p$, then $\bar{A}_{p}=\bar{A}$. Suppose $b$ is a point of $\bar{A}_{p}-\bar{A}$. Let $\varepsilon=d(b, \bar{A})$. Choose an Effros number $\delta$ for $\varepsilon$. Let $a$ be a point of $A$ such that $d(a, p)<\delta$. Let $h$ be an $\varepsilon$-homeomorphism of $M$. onto $M$ such that $h(p)=a$. Since $h(\mathrm{~b})$ belongs to $\bar{A}$, this is a contradiction. $A$ symmetric argument shows $\bar{A}$ is a subset of $\bar{A}_{p}$.

Property 2. If $\bar{A}$ is a proper subcontinuum of $M$, then $\bar{A}$ is homogeneous and acyclic. Let $\mathscr{G}$ be the collection of closures of arc components of $M$. Clearly $\mathscr{G}$ satisfies condition $\left({ }^{*}\right)$. Property 1 implies that $\mathscr{G}$ is a partition of $M$. Hence the hypotheses of Theorem 1 are satisfied, and Property 2 follows.

Property 3. No subcontinuum of $M$ is a simple closed curve. If the proper subcontinuum $K$ of $M$ were a simple closed curve, then $K$ would be a (closed) arc component of $M$ because $M$ is atriodic. Since $K$ would not be acyclic, this would violate Property 2.

Property 4. No arc in $\bar{A}$ contains an open set of $\bar{A}$. If some arc in $\bar{A}$ contained an open set of $\bar{A}$, then the homogeneity of $M$ would imply that $\bar{A}$ is a 1 -manifold. The only compact, connected 1-manifold is a simple closed curve.

Property 5. Each arc component of $\bar{A}$ is the one-to-one, continuous image of the real line. The continuum $\bar{A}$ contains no simple closed curve and no triod.

Let $p$ and $q$ be two points of an arc component of $M$. The sum of all arcs in $M$ that have $p$ as an endpoint and contain $q$ is called a ray starting at $p$.

Property 6. If $p$ is a point of $A$, then $A$ is the sum of two rays $R_{1}$ and $R_{2}$ starting at $p$ such that $R_{1} \cap R_{2}=p$.

Property 7. If $p$ is a point of $A$, and if one of the rays $R$ 
starting at $p$ has the property that $\bar{R}=\bar{A}$, then the other ray starting at $p$ also has this property. Suppose $S$ is the other ray starting at $p$ and $q$ is a point of $A-\bar{S}$. Let $\varepsilon=d(q, \bar{S})$. Let $\delta$ be an Effros number for $\varepsilon / 2$. Let $[p, r]$ be an arc in $A$ containing $q$ in its interior such that $d(p, r)<\delta$. Let $h: M \rightarrow M$ be a homeomorphism such that $h(p)=r$ and $h$ moves no point as much as $\varepsilon / 2$. Since $q$ is not a point of $h(\bar{S})$, it follows that $h(S)$ is the ray starting at $r$ that does not contain $q$ and that $q$ is not a point of $\overline{h(S)}$. Hence $[p, r]$ contains an open set of $\bar{A}$ containing $q$. This contradicts Property 4 .

Property 8. If $p$ is a point of $A$ and if $S$ is a ray starting at $p$, then $\bar{S}=\bar{A}$. Let $q$ be a point of $A-\bar{S}$. Let $\varepsilon=d(q, \bar{S})$. Let $\delta$ be an Effros number for $\varepsilon / 2$. It follows from the proof of Property 7 that no arc $[p, r]$ of $A$ containing $q$ has the property that $d(p, r)<\delta$. Hence, if $T$ is the ray starting at $q$ and not containing $S$, then $d(\bar{S}, \bar{T}) \geqq \delta$. Hence $[p, q]$ contains an open set of $\bar{A}$.

Property 9. Each proper nondegenerate subcontinuum $K$ of $\bar{A}$ is an arc. Let $p$ be a point of $K$. Since each of the rays starting at $p$ is dense in $\bar{A}$, there is an arc $\left[q_{1}, q_{2}\right]$ containing $p$ the endpoints of which are not in $K$. If $r$ were a point of $K-\left[q_{1}, q_{2}\right]$, then there would be an arc $[r, s]$ such that $s$ is not a point of $K$. Then $K \cap\left[q_{1}, q_{2}\right] \cap[r, s]$ would contain a triod.

Property 10. $\bar{A}$ is a solenoid, and $\bar{A}=M$. Hagopian [8] has shown that a homogeneous continuum with only arcs as proper nondegenerate subcontinua is a solenoid. Since a solenoid is not acyclic, Property 2 implies $\bar{A}=M$.

This completes the proof of the theorem.

Corollary (Bing). A simple closed curve is the only homogeneous plane continuum that contains an arc.

Proof. If $M$ is indecomposable, then $M$ is atriodic, for otherwise each composant of $M$ would contain a triod, contradicting the fact that the plane does not contain uncountably many disjoint triods.

If $M$ is a decomposable continuum that is not a simple closed curve, then $M$ admits a continuous decomposition into indecomposable, homogeneous continua, by Theorem 2 . No arc of $M$ can contain two points that lie in distinct decomposition elements [11]. Hence an arc in $M$ lies in a homogeneous, indecomposable subconti- 
nuum of $M$, and the previous paragraph may be applied to complete the proof.

Bing's paper [2] contains a powerful method of proof for homogeneous, plane continua. Hagopian, for instance, followed this method during part of his proof [7] that an indecomposable, homogeneous plane continuum is hereditarily indecomposable (so did Jones [10] in his announcement of the theorem some years earlier).

The outstanding problem in decompositions of homogeneous continua seeks to circumvent this argument. Posed by Jones sometime ago, the problem is the following.

Problem. Assume $M$ is an indecomposable, homogeneous plane continuum that contains a decomposable subcontinuum. Find a decomposition of $M$ into continua such that the resulting quotient space is a homogeneous, plane continuum that contains an arc.

Such a quotient space must be a simple closed curve, but this would contradict the indecomposability of $M$.

Another interesting problem, raised by Bing [2], is related to Theorem 3.

Problem. Does there exist a tree-like homogeneous continuum that contains an arc?

2. A relative version of the decomposition theory. This section contains a theorem for decomposing a closed subset of a homogeneous continuum. Additionally, it draws full generality in its conclusions and does not require all homeomorphisms to satisfy condition $(*)$.

THEOREM 4. Let $G$ be a partition into closed sets of the closed subset $K$ of the homogeneous compactum $M$. Let $\varepsilon>0$, and let $H$ be the set of $\varepsilon$-homeomorphisms of $M$. Suppose $H$ satisfies the following condition:

$$
\begin{aligned}
& \left(^{*}\right) \text { if } G, G^{\prime} \in \mathscr{G}, h \in H \text {, then } \\
& h(G) \cap G^{\prime} \neq \phi \Longrightarrow h(G)=G^{\prime} \text {. }
\end{aligned}
$$

Then

(1) The decomposition $\mathscr{G}$ is continuous,

(2) The quotient map $\pi: K \rightarrow N$ of $K$ onto the quotient space $N$ is completely regular

(3) Each component of each $G$ in $\mathscr{G}$ is homogeneous

(4) If $\operatorname{dim} K=m$ and $\operatorname{dim} N>0$, then $H^{m}(G)=0$, for some $G$ in $\mathscr{G}$, 
(5) If $\operatorname{dim} K=1$ and $\operatorname{dim} N>0$, then some component of some $G$ in $\mathscr{G}$ is hereditarily unicoherent and indecomposable,

(6) If each $G$ is homeomorphic to the discrete space $Z$, then $(K, \pi, N)$ is a locally trivial fiber space, and

(7) If each $G$ is homeomorphic to the discrete space $Z$ and $N$ is contractible, then $K$ is homeomorphic to the direct product $N \times G$.

Moreover, if either $K$ is a continuum or $H$ is the group of homeomorphisms of $M$, then

(8) The components of each $G$ in $\mathscr{G}$ are homeomorphic each to the other, and

(9) If $G, G^{\prime}$ belong to $\mathscr{G}$, then $G$ is homeomorphic to $G^{\prime}$. Finally, if $M=K$, then

(10) $N$ is homogeneous.

Proof. Most of the conditions can be proved by mimicing the proof of Theorem 1. Condition 4 follows from [15, Theorem 1]. Conditions 6 and 7 follow from [4, p. 109].

CoRollary (Hagopian). No homogeneous plane continuum other than a simple closed curve contains a hereditarily decomposable subcontinuum.

Proof. Let $X$ be a homogeneous plane continuum. Proceed as in $[6$, p. 38] to find a decomposable subcontinuum $K$ of $X$ and a monotone map $k: K \rightarrow[0,1]$. Let $0<r<t<1$. Define $\varepsilon=\rho\left(k^{-1}([r, t])\right.$, $\left.k^{-1}(0) \cup k^{-1}(1)\right)$. Let $\mathscr{G}=\left\{k^{-1}(s): r \leqq s \leqq t\right\}$, and let $H$ be the set of $\varepsilon$-homeomorphisms of $X$. The verification of (*) is similar to the proof in [6, paragraphs 4-6].

\section{REFERENCES}

1. R. H. Bing, A homogeneous indecomposable plane continuum, Duke Math. J., 15 (1948), 729-742.

2. - A simple closed curve is the only homogeneous bounded plane continuum that contains an arc, Canad. J. Math., 12 (1960), 209-230.

3. R. H. Bing and F. B. Jones, Another homogeneous plane continuum, Trans. Amer. Math. Soc., 90 (1959), 171-192.

4. E. Dyer and M. E. Hamstrom, Completely regular mappings, Fund. Math., 45 (1958), 102-118.

5. E. G. Effros, Transformation groups and $C^{*}$-algebras, Ann. of Math., (2) 81 (1965), 38-55.

6. C. L. Hagopian, Homogeneous plane continua, Houston J. Math., 1 (1975), 35-41.

7. - Indecomposable homogeneous plane continua are hereditarily indecomposable, Trans. Amer. Math. Soc., 224 (1976), 339-350.

8. - A characterization of solenoids, Pacific J. Math., 68 (1977), 425-435.

9. C. L. Hagopian and J. T. Rogers, Jr., A classification of homogeneous, circle-like continua, Houston J. Math., 3 (1977), 471-474. 
10. F. B. Jones, Homogeneous plane continua, Proc. Auburn Topology Conf., Auburn University, 1969.

11. - On a certain type of homogeneous plane continuum, Proc. Amer. Math. Soc., 6 (1955), 735-740.

12. - Certain homogeneous unicoherent indecomposable continua, Proc. Amer. Math. Soc., 2 (1951), 355-859.

13. J. T. Rogers, Jr., Completely regular mappings and homogeneous, aposyndetic continua, Canad. J. Math., 33 (1981), 450-453.

14. - Solenoids of pseudo-arcs, Houston J. Math., 3 (1977), 531-537.

15. D. C. Wilson, Completely regular mappings and dimension, Bull. Amer. Math. Soc., 76 (1970), 1057-1061.

Received March 14, 1980. The author was partially supported by a research grant from the Tulane University Senate Committee on Research.

TULANE UNIVERSITY

New ORLEANS, LA 70118 


\section{PACIFIC JOURNAL OF MATHEMATICS}

\section{EDITORS}

DONALD BABBITT (Managing Editor)

University of California

Los Angeles, California 90024

\section{Hugo Rossi}

University of Utah

Salt Lake City, UT 84112

C. C. MOore and Arthur AGuS

University of California

Berkeley, CA 94720
J. DugundJI

Department of Mathematics University of Southern California Los Angeles, California 90007

R. FinN and J. Milgram Stanford University Stanford, California 94305

ASSOCIATE EDITORS
R. ARENS
E. F. BECKENBACH
B. H. NeumanN
F. WOLF
K. YOSHIDA

\section{SUPPORTING INSTITUTIONS}

UNIVERSITY OF ARIZONA

UNIVERSITY OF BRITISH COLUMBIA

CALIFORNIA INSTITUTE OF TECHNOLOGY

UNIVERSITY OF CALIFORNIA

MONTANA STATE UNIVERSITY

UNIVERSITY OF NEVADA, RENO

NEW MEXICO STATE UNIVERSITY

OREGON STATE UNIVERSITY
UNIVERSITY OF OREGON

UNIVERSITY OF SOUTHERN CALIFORNIA

STANFORD UNIVERSITY

UNIVERSITY OF HAWAII

UNIVERSITY OF TOKYO

UNIVERSITY OF UTAH

WASHINGTON STATE UNIVERSITY

UNIVERSITY OF WASHINGTON 


\section{Pacific Journal of Mathematics}

Vol. 99, No. $1 \quad$ May, 1982

Mariano Giaquinta, Jindrich Necas, O. John and J. Stará, On the

regularity up to the boundary for second order nonlinear elliptic systems . . 1

Siegfried Graf, Realizing automorphisms of quotients of product $\sigma$-fields . . 19

Alfred Washington Hales and Ernst Gabor Straus, Projective colorings . . . 31

Sandra Hayes, The weak Nullstellensatz for finite-dimensional complex

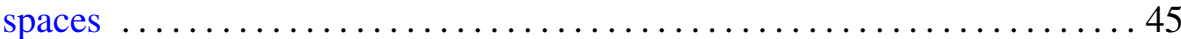

Gerald Norman Hile and Murray Harold Protter, The Cauchy problem

and asymptotic decay for solutions of differential inequalities in Hilbert

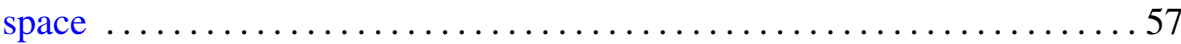

Robert D. Little, Projective space as a branched covering with orientable

branch set ......................................... 89

Jaroslav Mach, On the proximinality of Stone-Weierstrass subspaces . . . . . 997

John C. Morgan, II, On product bases ...................... 105

K. Balakrishna Reddy and P. V. Subrahmanyam, Altman's contractors

and fixed points of multivalued mappings . .................. 127

James Ted Rogers Jr., Decompositions of homogeneous continua . . . . . . . 137

Ahmed Ramzy Sourour, Characterization and order properties of

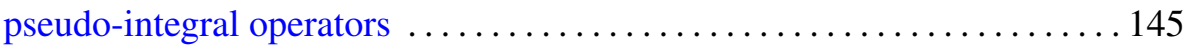

Robert Moffatt Stephenson Jr., Pseudocompact and Stone-Weierstrass

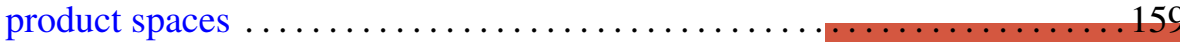

Bruce Stewart Trace, On attaching 3-handles to a 1-connected

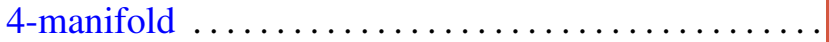

Akihito Uchiyama, The construction of certain BMO functions and the corona problem

Thomas Alva Whitehurst, An application of orthogonal polynomials to random walks ..............................

David J. Winter, Root locologies and idempotents of Lie and nonassociative algebras

William Robin Zame, The classification of uniform algebras on plane domains 